(2) Open Access Full Text Article

REVIEW

\title{
Approaches to the Diagnosis and Management of Ovarian Cancer in Pregnancy
}

This article was published in the following Dove Press journal:

Cancer Management and Research

\section{Kaja Michalczyk (10) \\ Aneta Cymbaluk-Płoska}

Department of Gynecological Surgery and Oncology of Adults and Adolescents, Pomeranian Medical University, Szczecin, Poland
Correspondence: Kaja Michalczyk

al. Powstańców Wielkopolskich 72,

Szczecin, 70-1II, Poland

Tel +48 9I 466 I332

Fax +48 9l 466 I334

Email kajamichalczyk@wp.pl
Abstract: Ovarian cancer is one of the most common gynecological cancers diagnosed in pregnancy. Its management is often very problematic due to the proximity of the adnexa to the developing fetus and chemotherapy-related toxicity risk. Tumor markers and imagining studies play important roles in diagnosis, help differentiate benign masses from malignancy and allow to plan the treatment. Due to the physiological changes that occur in pregnancy, levels of tumor markers can be altered and reduce their diagnostic value. We review current recommendations for the management and treatment of ovarian cancer in pregnant patients considering gestational age at diagnosis, tumor histology, stage of the disease, risk of obstetrical complications, and patient's preferences.

Keywords: pregnancy, chemotherapy, ovarian malignancy, tumor markers

\section{Introduction}

Pregnancy is a special period in every woman's life. Females often do not follow gynecological recommendations and skip routine check-up visits. Upon discovering the pregnancy, females sometimes undergo first gynecological examination and start routine pregnancy check-up control visits as they care for both themselves and the baby.

In pregnancy three ultrasound scans, one each trimester, are recommended. During routine obstetrical ultrasound examinations, adnexal masses are often incidentally discovered with most of them discovered during the first trimester. Only $3-6 \%$ of these are malignant and usually discovered at an early stage. ${ }^{1-3}$ Most of the adnexal masses are asymptomatic and regress in a spontaneous manner. If malignant, more than $50 \%$ tend to be symptomatic and present with pain, bleeding, dystocia, ovarian rupture or torsion. ${ }^{4}$

Cancer is a relatively rare finding during pregnancy as it occurs approximately in $0.05 \%$ to $1 \%$ of all pregnancies. Literature reports good oncologic and fetal outcomes in women treated for cancer during pregnancy. ${ }^{5,6}$ Statistically, ovarian cancer is the fifth most common cancer diagnosed during pregnancy, following breast, thyroid, cervical cancer and Hodgkin lymphoma. ${ }^{7}$ Ovarian cancer is often associated with poor prognosis, with $35 \%$ of 10 -year survival, while adolescent and young adults have as much as $85 \%$ chances of 10 -year survival. This may be associated with more favorable tumor histology as well as patients often being diagnosed in lower stage disease. ${ }^{8}$

Management of pregnant women with adnexal masses is difficult as many complications may occur causing danger both to the mother and the developing fetus. Ovarian 
cancer during pregnancy carries numerous challenges as it affects reproductive organs where the fetus develops A general recommendation is that, if the pelvic peritoneum and the pouch of Douglas cannot be reliably examined during surgery because of the enlarged uterus and limited possibility to manipulate it, restaging surgery should be planned postpartum. In patients who are considering pregnancy preservation, a cystectomy or an adnexectomy should be performed, followed by platinum-based chemotherapy and cytoreductive surgery after delivery, as surgery to no residual disease cannot be performed during pregnancy. Cancer treatment during pregnancy must balance between maternal and fetal health. There is no evidence suggesting adverse effects of pregnancy on the survival of women with ovarian cancer. ${ }^{9}$

In order to obtain the best possible outcome, an adequate and appropriate treatment is required, while considering its risks to the pregnancy and a developing fetus. With the use of imagining such as ultrasound and clinical markers, accurate differentiation between benign and malignant adnexal masses is crucial to decide on further actions and management. As demonstrated in Figure 1, a multidisciplinary approach including a variety of specialists should be proposed to obtain the best model of care of a pregnant patient diagnosed with cancer.

Although epithelial cancer is the most common cancer outside of pregnancy and accounts for almost $90 \%$ of all

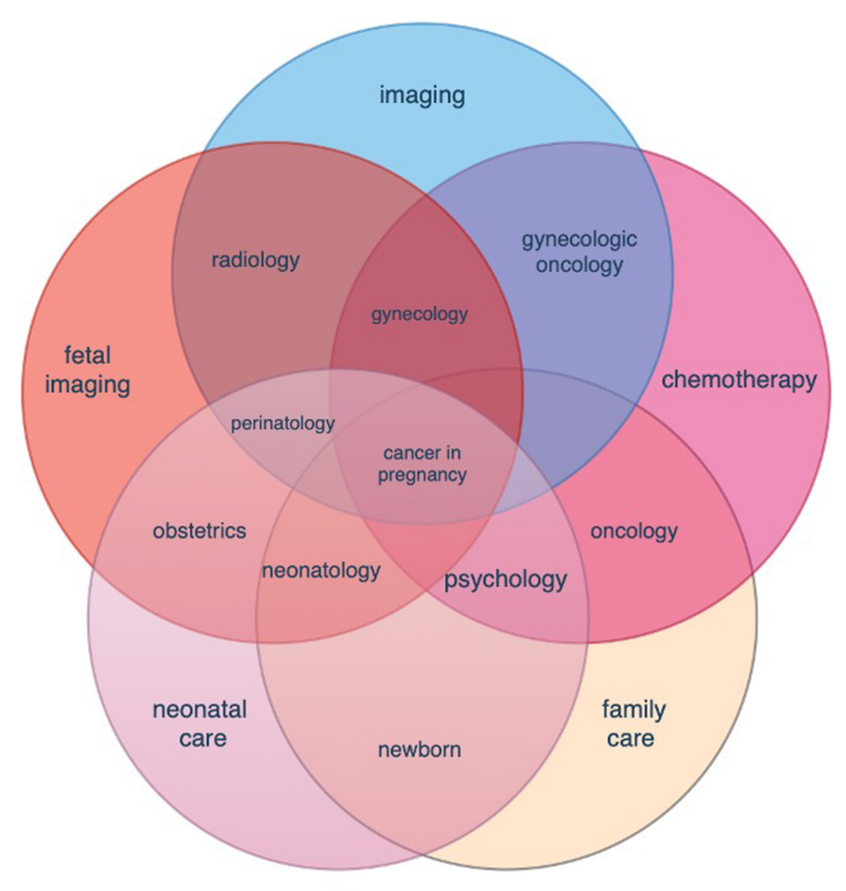

Figure I Venn diagram illustrating the management of patients diagnosed with ovarian cancer in pregnancy. ovarian cancers, during pregnancy germ cell tumors occur at a highest frequency. ${ }^{10}$ Non-epithelial cancers, including germ cell and stromal cancers, tend to present with bulky masses, reaching high dimensions. Patients are often symptomatic and present with pain, abdominal distension or bleeding. Due to the frequency of ultrasounds in pregnancy, approximately $90 \%$ of pregnant patients with ovarian cancer are diagnosed with stage I disease.

Treatment of epithelial ovarian cancer should consist of a combination of surgery and chemotherapy. Surgery can be performed either via laparotomy or laparoscopy. If necessary, secondary surgery can be undertaken following the delivery. Patients with advanced stage disease should undergo debulking surgery. Complete cytoreduction may be impossible in presence of the fetus and may cause unnecessary risks to the mother and the fetus. ${ }^{5}$ Initiation of chemotherapy treatment during pregnancy is a possible strategy to improve maternal outcome while preserving the pregnancy and delaying the delivery. ${ }^{11}$

In pregnancy, the surgery should include removal of the ovaries and peritoneal staging with preservation of the uterus. Only potentially suspicious lymph nodes should be removed to minimize the risk associated with the surgery. Depending on the term of pregnancy, after multidisciplinary consultations, patients with peritoneal spread could be proposed with pregnancy termination, premature delivery or neoadjuvant chemotherapy and preservation of the pregnancy. $^{12}$

This review provides insight into current diagnostic and treatment methods for pregnant patients diagnosed with ovarian cancer. For the purpose of our review, we conducted a literature search on Pubmed, Web of Science, and Cochrane Library databases including studies up to October 2020. We evaluated the information provided in articles published in English using a combination of keywords relevant to ovarian cancer, chemotherapy, surgical treatment and pregnancy. It contributes to this research area by providing evidence-based information on currently used diagnostic and therapeutic methods. Possible complications that may occur at different timing of the pregnancy were evaluated. Even though ovarian cancer diagnosis during pregnancy is a rare finding, the clinical management is difficult as it affects both the mother and the developing fetus.

\section{Epidemiology}

Epithelial ovarian cancer (EOC) is the most aggressive histology type of ovarian cancer. It is the most common 
form among non-pregnant women. The overall prognosis of patients with epithelial cancer is worse than that of nonepithelial cancer. Peritoneal and nodal spread is usually present and patients require adjuvant chemotherapy (in early-stage or debulked advanced stage disease) or neoadjuvant treatment (in advanced stage or unresectable peritoneal disease). ${ }^{12}$ In Table 1 we present the most frequent histological types of ovarian cancer diagnosed in pregnant women.

\section{Diagnostics}

\section{Radiological Examination}

Imaging studies are important tools in diagnostic and evaluation of acute and chronic medical conditions. Concerns about the safety of these procedures during pregnancy sometimes result in fear and avoidance among patients. Ultrasonography and magnetic resonance imaging are the studies of choice during pregnancy as they do not carry any associated risks neither to the mother or fetus; They should be only used when necessary to provide medical benefit to the patient. ${ }^{14}$

Pelvic ultrasound is a primary, cheap and easily available test for diagnosis and evaluation of an adnexal mass. Findings that may suggest a malignant potential of an

Table I Most Frequent Histological Types of Borderline and Malignant Adnexal Masses in Pregnancy and Their Management

\begin{tabular}{|c|c|c|}
\hline Prevalence & Histological Type & Histological Subtype \\
\hline $48.1 \%$ & Borderline & $\begin{array}{l}\text { Serous, endometrioid, } \\
\text { mucinous }\end{array}$ \\
\hline \multirow[t]{6}{*}{$21.6 \%$} & \multirow[t]{6}{*}{ Epithelial tumors } & Clear cell \\
\hline & & Endometrioid \\
\hline & & Low grade serous \\
\hline & & Mucinous \\
\hline & & High grade serous cancer \\
\hline & & $\begin{array}{l}\text { Carcinosarcoma/ } \\
\text { undifferentiated/other }\end{array}$ \\
\hline \multirow[t]{3}{*}{$24.6 \%$} & \multirow[t]{3}{*}{ Germ cell tumors } & Dysgerminoma \\
\hline & & Yolk sac \\
\hline & & Immature Teratoma \\
\hline \multirow[t]{2}{*}{$0.5 \%$} & \multirow{2}{*}{$\begin{array}{l}\text { Sex cord stromal } \\
\text { tumors }\end{array}$} & Sertoli, Lydig Tumor \\
\hline & & Granulosa cell tumor \\
\hline
\end{tabular}

Note: Based on the data puiblished by Aggarwal and Kehoe, ${ }^{4}$ Lockley et al, ${ }^{8}$ and Cavaco-Gomes et al. ${ }^{13}$ adnexal mass are the following: tumor size $>10 \mathrm{~cm}$, speed of growth $>0.35 \mathrm{~cm} /$ week, presence of solid areas, multiloculated masses, gross internal septa, papillary projections, bilateral masses, increased vasculature, presence of free abdominal-pelvic fluid, or ascites. ${ }^{10,15}$ In order to improve the accuracy of ultrasonography and to gain additional characteristics, color doppler imaging can be used to obtain a vascular map of the adnexal mass. ${ }^{16}$

If ultrasonography is ambiguous or insufficient further imaging studies may be conducted using magnetic resonance. MRI provides the ability to imagine deep soft tissue structures without using ionizing radiation, making it safe during pregnancy. It is especially useful for evaluation of big masses, their relationship with the surrounding structures and their infiltration. In patients with an advanced stage disease, it allows to evaluate the retroperitoneal space and lymph nodes involvement. The use of gadolinium contrast with MRI is controversial and should be limited as it is a water-soluble agent and can potentially cross the placenta and enter the amniotic fluid and fetal circulation. ${ }^{17}$ DWI (diffusion weighted imaging) is recommended.

Current imaging studies such as radiography, computed tomography scans and nuclear medicine imagining techniques are conducted at a much lower dose from the exposure associated with fetal harm. If necessary, they should not be withheld from a pregnant patient. ${ }^{14}$ It should be remembered that imaging studies cannot give a clear answer, whether the change is malignant or not. The determination of tumor markers seems to be even more important during pregnancy than outside of it and enables to decide on further patient's management. If necessary, for example to exclude distant lung metastases, X-ray imaging can be performed in pregnant women using pelvic shielding to lower the dose of radiation to the uterus and the developing fetus.

\section{Tumor Markers}

Many serum markers are used to aid the diagnosis of ovarian cancer. In Table 2 we list the commonly used markers in patients presenting with adnexal masses. We also evaluate their changes in pregnancy.

CA-125 is a tumor marker associated with EOC widely used in non-pregnant patients.

During pregnancy, it is produced by the decidua and granulosa cells. ${ }^{12}$ All studies describing Ca 125 levels in individual trimesters report an increase of about $50 \%$ in the first trimester (median $59.5 \mathrm{U} / \mathrm{mL}$ ), ${ }^{18}$ systematic recovery in the second trimester $(27.8 \mathrm{U} / \mathrm{mL})$ and minimal 
Table 2 Ovarian Tumor Markers and Their Changes During Pregnancy

\begin{tabular}{|c|c|c|c|}
\hline $\begin{array}{l}\text { Histological Type of } \\
\text { Ovarian Tumor }\end{array}$ & $\begin{array}{l}\text { Tumor } \\
\text { Marker }\end{array}$ & Normal Range & Changes in Pregnancy \\
\hline \multirow[t]{3}{*}{ Epithelial tumor } & CA 125 & $\leq 35 \mathrm{U} \mathrm{mL}^{-1}$ & Increased in Ist trimester, then decreases \\
\hline & CEA & $\leq 5 \mathrm{ng} \mathrm{mL}^{-1}$ & Not influenced by pregnancy \\
\hline & $\mathrm{He} 4$ & $\leq 70 \cup \mathrm{mL}^{-1}$ & Lower levels in pregnant women, mildly increased values in $3 r d$ trimester \\
\hline Mucinous tumor & CA 19.9 & $\leq 37 \cup \mathrm{mL}^{-1}$ & Mildly increases with increasing gestational age; never exceeds normal range \\
\hline Mucinous carcinoma & Inhibin A & $\leq 17.3 \mathrm{pg} \mathrm{mL}^{-1}$ & Increased values in Ist trimester; abnormally increased in Down Syndrome \\
\hline Granulosa cell tumor & Inhibin B & $\leq 255 \mathrm{pg} \mathrm{mL} L^{-1}$ & Not influenced by pregnancy \\
\hline \multirow[t]{2}{*}{ Germ cell tumors } & b-HCG & $\leq 0.5 \mathrm{mlU} \mathrm{mL}^{-1}$ & Physiologically increased in pregnancy \\
\hline & AFP & $\leq 10 \mathrm{ng} \mathrm{mL}^{-1}$ & $\begin{array}{l}\text { Physiologically increased in pregnancy; abnormally increased in neural tube } \\
\text { defects; decreased in Down Syndrome }\end{array}$ \\
\hline Dysgerminoma & LDH & $\leq 250 \cup \mathrm{mL}^{-1}$ & Increased in severe preeclampsia, HELLP syndrome \\
\hline
\end{tabular}

Note: Based on the data published by Cavaco-Gomes et al. ${ }^{13}$

growth in the third trimester (median $31.3 \mathrm{U} / \mathrm{mL}$ ). Nevertheless, this marker seems to be of little use due to the excessive possibility of false-positive results, which would result in inappropriate qualification of patients to the group of high risk of ovarian cancer.

He4 appears to be a marker whose concentration is more stable throughout the pregnancy. The median of $36.9 \mathrm{pmol} / \mathrm{L}$ in the first trimester versus the second trimester $-39.8 \mathrm{pmol} / \mathrm{L}$ and third trimesters $-54.6 \mathrm{pmol} / \mathrm{L}$ did not differ significantly. The concentration of HE4 protein throughout pregnancy remains within the reference values and is significantly lower than in postmenopausal women. ${ }^{19}$ The data presented by $\mathrm{Lu}$ et $\mathrm{al}^{18}$ showed that the ROMA algorithm (due to the increased values of the Ca 125 marker) misclassified patients, respectively: 70.6\% in the first trimester, $4 \%$ in the second trimester and $42.9 \%$ in the third trimester. It seems that the usefulness of the ROMA algorithm has a little use in both the first and third trimesters. In summary, the most diagnostic epithelial marker during pregnancy seems to be the $\mathrm{He} 4$ marker and this is what we should suggest when deciding on a surgical procedure.

The second group of ovarian cancers that affect pregnant women is the non-epithelial cancer of the ovary. Dysgerminoma, being one of them is the most common cancer diagnosed in pregnancy. The most widely used marker in the diagnosis of dysgerminoma is lactate dehydrogenase (LDH). Its concentration in a normal pregnancy, as reported by Makonnen et al does not change. ${ }^{20} \mathrm{LDH}$ activity is increased in women with chronic arterial hypertension, severe pre-eclampsia and during both physiological and pathological labor. Lactate dehydrogenase, which is a glycolytic enzyme, has 5 isoenzymes, of which two fast LDH-1 and LDH-2 seem to be useful not only for the diagnosis of dysgerminoma but also for monitoring its treatment. $^{21}$

Another marker is AFP; maternal AFP level in pregnancy begins to increase from week 14 of pregnancy to week 32 of pregnancy. First, it is produced by the yolk sac, then by the fetal liver and gastrointestinal tract. Maternal serum AFP level is used to screen for certain fetal abnormalities, particularly neural tube defects and trisomy 21 . Nevertheless, significantly elevated levels of AFP appear to be always associated with endodermal sinus tumors. Many reports emphasize the fact that germ-cell tumor, and above all yolk suck tumor, should be considered as the cause of elevated serum AFP concentration in pregnant women in the absence of fetal malformations or maternal disease. $^{22,23}$

Beta hCG (chorionic gonadotropin) is a hormone produced mainly during pregnancy and controls its development. Disturbances in beta hCG secretion lead to a number of complications and indicate abnormalities in the course of pregnancy. We must remember that beta $\mathrm{hCG}$ is one of the five members of the hCG family. Sulfated hCG and hCG are hormones produced by placental syncytiotrophoblast cells and pituitary gonadotropin cells. Hyperglycosylated hCG is an autocrine factor produced 
by placental cytotrophoblast cells, which is a sign of malignancy in placental cancer. ${ }^{24,25}$ It seems that the usefulness of this marker during pregnancy is highly questionable and is not used in everyday clinical practice.

The most common gonadal neoplasm in pregnant women is granulosa cell tumor. The diagnostic sensitivity of inhibin $\mathrm{A}$ in non-pregnant women with granuloma is $67 \%$ and the specificity is $100 \%$. In contrast, in the case of inhibin $\mathrm{B}$, the sensitivity is $89 \%$ and the specificity is $100 \%$. Inhibin A is the dominant molecular form of inhibin in maternal circulation from week 4 of pregnancy. Although the exact biological function of inhibin $\mathrm{A}$ in pregnancy is not fully understood, recent studies suggest that inhibin A may be a more useful marker of placental function than beta hCG due to its shorter half-life. It is currently believed that monitoring inhibin A levels in early pregnancy may help predict miscarriage, Down syndrome, pre-eclampsia and IUGR in both the first and second trimesters of pregnancy. Its use in the diagnosis of ovarian tumors is not recommended. ${ }^{26}$ Inhibin B concentrations, physiologically during pregnancy, decrease sharply in the first trimester and increase slightly in the second trimester. The usefulness of inhibin B for the diagnosis and monitoring of gonadal tumors in pregnancy, especially granulosa juvenile, seems to be justified. $^{27}$

The determination of LDH may be used in germinal tumors, eg, dysgerminoma, to monitor the effectiveness of the treatment and possible recurrence of the malignancy, in patients in whom the marker was increased before the primary diagnosis and surgery. The values of any marker, not only $\mathrm{LDH}$, should not influence the decision regarding the extent of surgical treatment. In young patients, in whom a germinal or rarely gonadal tumor (juvenile folliculoma) is suspected based on the results of imaging tests, a high baseline level of the Ca 125, HE4, AFP, LDH or Beta HCG could allow monitoring of the treatment process and early detection of possible recurrence.

In case of germinal tumors, the use of specific markers is low (with the extent of a marked increase of AFP in yolk sac tumor) as they only rise in approximately $50-60 \%$ of patients.

The decision regarding fertility sparing surgery should be made only on the basis of the patient's clinical advancement. Neoadjuvant chemotherapy is mainly used in patients with high clinical advancement of epithelial ovarian cancer. As for germinal ovarian neoplasms, the prognosis is so good that a sparing treatment with optional adjuvant chemotherapy is preferred depending on the patient's staging.

Currently, there is undergoing clinical research for use of Anti-Mullerian Hormone (AMH) in the diagnosis of gonadal tumors in pregnancy. Thanks to reports by Nelson and La Marca, ${ }^{28,29}$ it is known that AMH levels decline in the second and third trimesters of pregnancy, with normal levels in the first trimester. Its usefulness seems to be highly probable. Subsequent publications will bring us closer to the possibility of using AMH both in the diagnosis and monitoring of ovarian gonadal tumors.

\section{Treatment}

\section{Surgical Management}

In case of a low risk of suspicion of malignancy, the surgery should be performed in an elective mode, planned by oncological gynecologists, obstetricians and neonatologists, while if the possibility of malignancy, based on clinical imaging features (i.a. solid consistency, papillary projections) and elevated markers, there is no reason to delay until second trimester. If both ovaries are removed prior to 10-12 weeks progesterone should be given to support corpus luteum. Whenever possible, it should be performed during the safest period of pregnancy, which is the second trimester of pregnancy (16-18 weeks of pregnancy). It is the time, when the risk of spontaneous miscarriage and the hormonal dependence of the corpus luteum is reduced. Moreover, by this time, most of the functional cysts will resolve spontaneously. ${ }^{13,30,31}$ Literature suggests elective surgical management of patients with ovarian cancer as emergency surgical interventions result in higher rates of adverse effects including miscarriage, premature rupture of membranes or delivery. $^{32}$

If the adnexal mass was noticed in the third trimester and the suspicion of malignancy is low, waiting for fetal maturity is recommended; in case of surgical exploration, there is a risk of premature delivery. Furthermore, the surgery in a more advanced pregnancy is often technically more difficult as both the uterus and fetus are larger, decreasing the surgical field, and the risk of adverse obstetric outcomes is higher. "Emergency surgery" should be performed in symptomatic patients presenting with enlarging tumor size, strong suspicion of malignancy, severe clinical manifestations including hydronephrosis, and sudden events such as vomiting, rupture or hemorrhage. 
Due to an increased risk of preterm labor, steroids should be administered between 24 and 36 weeks of gestation to minimize the risk of respiratory distress syndrome, neonatal death, and intraventricular bleeding. ${ }^{10}$ Tocolysis is not recommended and should only be considered in the event of postoperative uterine contractions. ${ }^{33}$

Laparoscopy in pregnancy is feasible, but depends on the gestational age, surgeon's experience, type of procedure and the organs of interest. The procedure should be carried in accordance with SAGES (Society of American Gastrointestinal and Endoscopic Surgeons) recommendations. ${ }^{33}$ The benefits of laparoscopic surgery include lower perioperative pain, less bleeding, early upright standing, reduced risk of thromboembolism, faster return of peristalsis, shorter hospitalization time, faster return to daily activities, less risk of surgical wound infection, less manipulation of the uterus and minimized risk of preterm delivery. Laparoscopic management is also associated with the following potential problems: placental perfusion can be impaired due to high abdominal pressure. Moreover, maternal conversion of carbon dioxide to carbonic acid can cause fetal acidosis. Lastly, the pregnant uterus may be injured when inserting a Veress needle or trocars. In the management of pregnant patients' lower amounts of carbon dioxide can be insufflated and intraoperative monitoring including capnography can be used.

On the other hand, the advantages of laparotomy include better accessibility to the epigastric region and adnexa, ability to evaluate lymph nodes in palpation, lack of $\mathrm{CO} 2$ inflation resulting in better perfusion and placental circulation, no need of Trendelenburg positioning. Microlaparotomy can be a safe alternative. To improve placental blood flow, the patient could be positioned in left lateral tilt during surgery.

The choice of treatment approach should depend on the tumor size, its morphological appearance (on radiography and during surgical exploration), histological subtype, extent of the disease, timing of pregnancy (term) and patient's wishes. ${ }^{12}$

If performed laparoscopically, the procedure should be done with standard precautions to avoid the spread of malignant cells (particularly from tumor rupture). In patients with suspicion of malignant lesion on imaging, the procedure should include salpingo-oophorectomy of the affected side and peritoneal cytology. Intraoperative histological analysis of frozen-section specimen should be conducted in order to decide further management of the patient. ${ }^{12}$

\section{Fertility Sparing Surgery Epithelial Ovarian Cancer}

The possibility of fertility sparing surgery is especially important for young patients diagnosed in early stages of ovarian cancer. For many years, radical surgery was the procedure of choice even for patients diagnosed with early stage of the malignancy. However, approximately 40 years ago, the fertility sparing surgery was first implemented in patients with FIGO IA and surgeons started to investigate the possible risks and advantages of using this type of surgery. ${ }^{34}$ Kajiyama et $\mathrm{al}^{35}$ conducted a research on a large group of patients, they proved that there are no differences in either DFS or OS of patients with Low stage, ie, IA and IC operated with sparing or radical surgery. In the follow-up, there was no difference in overall survival in patients with IA and IC (ie, with a damaged capsule or tumor cells in the peritoneal fluid).

In case of clear cell carcinoma, the use of sparing surgery is only recommended for patients diagnosed with stage IA. ${ }^{36,37}$ In the case of patients with FIGO IB, in whom the disease affects both of the ovaries, the 2018 National Comprehensive Cancer Network (NCCN) guidelines recommend sparing only the uterus. Taking into account the psychological aspect and the young age of patients, conservative surgery could be offered in patients wishing to preserve their fertility. However, these young women need to be aware of the fact that in the case of fertility sparing surgery, if the clinical staging is advanced and/or the disease is already disseminated, the procedure should be radicalized. The decisions regarding patient's management are always difficult and should be discussed with a multidisciplinary team of specialists evolving gynecologists and oncologists. Patients should be always offered and provided with psychological support.

\section{Non-Epithelial Ovarian Cancer}

Germinal ovarian tumors in young patients are usually highly chemosensitive and sparing surgical treatment is recommended. In case of dysgerminoma, which is the most frequent embryonic tumor, as many as $70 \%$ of cases are diagnosed in stage IA and only $5 \%$ in stage IV. ${ }^{38}$ The treatment usually consists of unilateral removal of the appendages without the need for adjuvant chemotherapy. In only around $10 \%$ of patients, the second ovary is involved, therefore it is not currently recommended to take biopsies from the second ovary if the macroscopic appearance is normal. If both ovaries are involved, adnexectomies are performed on the side of the 
larger tumor and cystectomies on the opposite side. The prognosis for long-term survival is very good, recurrences occur depending on the clinical stage, up to $25 \%$, but the possibility of their cure is estimated at $90 \%{ }^{39}$ Yolk suck tumor is a germline cancer often associated with a poor prognosis. Before the BEP protocol era, a 5-year survival was around $15 \%$. Recently, sparing treatment with the need (except IA) of subsequent adjuvant chemotherapy is fully accepted. In 2015, Satoh examined as many as 211 patients and found that after using sparing treatment plus III BEP regimens, the 5-year survival was $93.4 \%{ }^{40}$ The situation is slightly different in case of gonadal tumors.

The greatest incidence of the juvenile form of tumor is at approximately 20 years of age. These tumors are not as highly chemosensitive as terminal tumors, however can be well managed with a radical surgery. In case of relapse, they are difficult to cure and often appear after many years from the primary diagnosis. Currently, fertility-preserving operations are allowed in patients presenting with FIGO IA and the treatment consists of removal of the adnexa on the tumor side and staging. There is no need to perform a biopsy of the other ovary. ${ }^{39}$ Likewise, sparing treatment is acceptable in the case of IA development for cords and genital stromal tumors. There are limited data on the follow-up of patients undergoing fertility sparing surgeries due to a low number of cases. There are several publications in the form of case studies that describe pregnancies after sparing surgeries. ${ }^{41,42}$ Summing up, modern medicine makes it possible to perform fertility-preserving surgery even in more aggressive types of cancer. Based on the clinical staging and histopathology of the malignancy, chemotherapy is often required as a complementary treatment.

\section{Systematic Treatment}

In Western and Central Europe, the age of women deciding to get pregnant has been increasing in recent years. Due to the more mature age of pregnant women, the number of non-epithelial and ovarian cancers is still increasing. Currently, a diagnosis of a malignant tumor in a pregnant woman does not mean a life sentence. There are more and more reports on a safe and effective use of anti-cancer drugs in pregnant women. Below we present management guidelines for pregnant women with differentiation into epithelial and non-epithelial ovarian tumors, with particular emphasis on the safety aspects of pharmacotherapy.
It should be remembered that the risk of teratogenicity of anti-cancer drugs depends not only on the type of drug but also on the time of its administration. The average time from 8 to 14 days after conception is commonly known as the "all or nothing" period. If teratogenic drugs act on the embryo during this time, this exposure may disrupt the processes that facilitate implantation and lead to miscarriage. If implantation occurs, it will be successful without affecting the proper development of the fetus. This is done thanks to the totipotent cells of the embryo that allow the repair and regeneration of damaged tissue. ${ }^{43}$ Summing up, the administration of chemotherapy drugs up to 2 weeks after conception, in the time, when patients usually do not yet suppose that they are pregnant, does not cause congenital malformations. The period of organogenesis from the 3 rd to the 8 th week of pregnancy seems to be the most critical period of exposure to drugs. The period of gastrulation from the 3 rd to 5 th week of pregnancy seems to be particularly sensitive, as there is the fastest differentiation of tissues and the most extensive and irreversible damage may occur. We must also remember that each organ has a different susceptibility to damage. There are structures in the body such as the CNS, genitals, eyes, hearing and hematopoietic system which are sensitive to teratogenic even after organogenesis is completed. It is recommended to start chemotherapy only after the end of the 14 th week of pregnancy. ${ }^{44}$ Due to the lack of studies on the pharmacokinetics of cytostatics in pregnant women, the same doses of drugs as in non-pregnant women calculated on the basis of the body surface are used. Some scientists have drawn attention to the fact that physiological changes occur during pregnancy that may affect drug distribution. For example, the volume of plasma increases, the amount of albumin decreases, and the elimination of toxins by the kidneys and liver increases. The use of chemotherapy during the second and third trimesters of pregnancy appears to be safe. There are reports that the administration of cytostatics in these trimesters increases the risk of intrauterine growth restriction (IUGR) and contributes to low birth weight in children. Between the last chemotherapy cycle and the delivery, a 3-week interval is recommended to prevent the hematopoietic suppression in mother and fetus caused by chemotherapeutic agents. ${ }^{45}$ Hematological toxicity can result in an augmented risk for infections and bleeding complications during delivery. ${ }^{46}$ The main message for the use of cytostatics during pregnancy is that the treatment of a patient with ovarian cancer is with an optimal treatment regimen 
without harm to the developing fetus. The treatment for EOC should consist of surgical staging and chemotherapy (in all patients apart from staging IA, grade 1-2). As in non-pregnant patients, the recommended chemotherapy regimen in pregnancy is carboplatin and paclitaxel. Neoadjuvant chemotherapy (NACT) should be administered in the advanced stages of the disease to maintain pregnancy. $^{12}$ Numerous reports on the use of taxanes, including docetaxel and paclitaxel during pregnancy indicate their low harmfulness to the fetus in the second and third trimesters. The exceptions were - the described case of pyloric stenosis and three cases of myelosuppression in children. Taxanes are substrates for the P-glycoprotein, which is present in high concentrations in the placenta. Taxanes are also highly metabolized by cytochrome P-450, the concentration of which increases in the third trimester of pregnancy by $50-100 \%$, which enables their relatively safe use during pregnancy. Complications following the use of this group of drugs include neurotoxicity as well as toxic effects on the digestive and respiratory systems. Van Calsteren et $\mathrm{al}^{11}$ conducted an animal study on the transplacental transfer of taxanes and found very low levels of taxanes in plasma and fetal tissues after administration of chemotherapy. Tissue concentrations remained detectable up to 72 hours after infusion. This is explained by the high solubility of taxanes in fats, their high molecular weight and the significant binding of taxanes with proteins, which makes it difficult to pass through the placenta. ${ }^{47}$ Only ex vivo data are available on the transplacental transfer of taxanes in humans. As demonstrated in a placental perfusion model, the transplacental transfer rate of paclitaxel was found to be low. Only one report documented a comparative transplacental transfer of both taxanes, and failed to demonstrate any significant difference between paclitaxel and docetaxel. ${ }^{48}$ Cardonick and Iacobucci ${ }^{49}$ reported absence of fetal complications in mothers receiving platinum-based chemotherapy. In pregnancy, exposure to carboplatin during the second and third trimesters does not appear to increase the risk of serious malformations. The action of platinum compounds is based on the formation of DNA adducts, which cause DNA cross-linking. DNA cross-linking inhibits replication, transcription, and other nuclear functions of cells. The combination of these events stops cell proliferation and ultimately tumor growth. Cisplatin and carboplatin are among the most commonly used platinum compounds. Due to their low molecular weight, they easily cross the placenta by diffusion. Large amounts are detected in the fetal plasma.
Cisplatin may be more nephrotoxic and ototoxic to the fetus. In contrast, carboplatin produces greater myelosuppression. Multiple studies have reported good oncologic and fetal outcomes when combination treatment with paclitaxel and carboplatin was used. ${ }^{4,50}$ In the treatment of non-epithelial cancers, surgical resection of the adnexal mass and staging are recommended. As in non-pregnant women, patients in advanced stages should undergo chemotherapy. The standard protocol is bleomycin, etoposide and platinum (BEP). ${ }^{12}$ Bleomycin is a chemotherapeutic agent also used in treatment for Hodgkin's Lymphoma. Its use during pregnancy was found to be associated with birth defects (ventriculomegaly, plagiocephaly, syndactyly, pectus excavatum), increased risk of IUGR and neonatal complications. In the era of coronavirus COVID-19, it is recommended to abandon the use of bleomycin, which causes pulmonary fibrosis and to use the EP regimen based on etoposide and cisplatin. $^{51}$ In case of nonepithelial ovarian tumors in pregnancy, many authors recommend as well to follow the routine ovarian cancer regimen which is paclitaxel and carboplatin. There were no inferior results with this regimen when considering recurrences and overall survival. ${ }^{52}$ Carboplatin is preferred for gynecological malignancies except for germ cell cancers, in which a cisplatin-based schedule is standard of care. Pediatricians should be made aware of possible ototoxicity even if newborn hearing screening normal. Etoposide remains relatively myelotoxic but its use during pregnancy in combination with cisplatin with or without bleomycin has been described and appears to be safe, although numbers of cases are limited. ${ }^{53-55}$

The chemotherapy dosage for systematic treatment should be based on patients' actual body weight and the dose $/ \mathrm{m}^{2}$ or dose $/ \mathrm{kg}^{2}$ should be used as in nonpregnant patients. $^{56}$

Based on the current ESMO recommendations, the use of anti VEGF and other antiangiogenic drugs is contraindicated during pregnancy. Moreover, until safety clinical data become available, targeted therapies should be avoided during pregnancy and breast feeding. ${ }^{56}$ The side effects differ based on the term of pregnancy in which they are administered. The use of immunotherapy in early pregnancy can increase the risk of miscarriage, while its administration during the second or third trimester can increase the risk of stillbirth, premature delivery and infant mortality. ${ }^{57}$ The use of angiogenesis inhibitors within the first weeks from conception can cause "all or none" effect thus the pregnancy will either continue or fail. In the first 
trimester it could cause an increased risk of miscarriage, skeletal malformations and abnormal vascular development of the fetal organs. From the second trimester onwards, the use of angiogenesis inhibitors was found to be associated with an increased risk of intrauterine growth restriction, preeclampsia and hypertension. ${ }^{58}$

\section{Follow-Up}

The most common complications associated with an adnexal malignancy during the pregnancy are: ovarian torsion and/or rupture, abortion and low birth weight and preterm delivery. The bigger the size of the tumor, the higher the risk of complications. ${ }^{59}$ One of the major concerns is impact of the administered chemotherapy on fetal well-being. A parameter that could potentially be monitored is fetal anemia by measuring peak systolic velocity (PSV). Halaska et al have demonstrated its potential use in monitoring fetal anemia. ${ }^{46}$ Using multiples of the median of PSV authors were able to predict the severity of fetal anemia. Significant obstetric risk is always present in emergency situations, eg, ovarian torsion. Rapid patient management in a referral multidisciplinary center is crucial for a successful outcome. Both maternal and fetal vital functions should be monitored. Decisions regarding the treatment should be undertaken after evaluation of term of pregnancy, fetal maturation and maternal parameters.

\section{Ethical Considerations}

Upon diagnosis, the following aspects should be raised by a multidisciplinary team and discussed with the patient:

1. Fetal loss or its prematurity

2. Worsening maternal oncologic outcomes

3. Potential effects on the fetus

4. Future fertility

As the incidence of ovarian cancer in pregnancy is low, still, there are no guidelines for patient's management. Pregnant patients have a similar outcome as non-pregnant women. Pregnancy should be preserved and continued whenever possible. A multidisciplinary team consisting of oncologists, psychologists, gynecologists, obstetricians, perinatologists and neonatologists should decide on the treatment strategy. Management of ovarian cancer depends on its histological subtype, staging, size of the tumor and the term of pregnancy. Iatrogenic prematurity should be avoided due to associated problems with preterm birth. Cancer treatment during pregnancy, both surgical and systematic is possible, and should adhere as much as possible to the standard protocol of care offered to non-pregnant patients.

\section{Disclosure}

The authors report no conflicts of interest for this work.

\section{References}

1. Mancari R, Tomasi-Cont N, Sarno MA, et al. Treatment options for pregnant women with ovarian tumors. Int J Gynecol Cancer. 2014;24 (6):967-972. Lippincott Williams and Wilkins. doi:10.1097/ IGC.0000000000000161

2. Zhao XY, Huang HF, Lian LJ, Lang JH. Ovarian cancer in pregnancy: a clinicopathologic analysis of 22 cases and review of the literature. Int J Gynecol Cancer. 2006;16(1):8-15. doi:10.1111/ j.1525-1438.2006.00422.x

3. Leiserowitz GS, Xing G, Cress R, Brahmbhatt B, Dalrymple JL, Smith LH. Adnexal masses in pregnancy: how often are they malignant? Gynecol Oncol. 2006;101(2):315-321. doi:10.1016/j. ygyno.2005.10.022

4. Aggarwal P, Kehoe S. Ovarian tumours in pregnancy: a literature review. Eur J Obstet Gynecol Reprod Biol. 2011;155(2):119-124. Elsevier Ireland Ltd. doi:10.1016/j.ejogrb.2010.11.023

5. Amant F, Halaska MJ, Fumagalli M, et al. Gynecologic cancers in pregnancy: guidelines of a second international consensus meeting. Int J Gynecol Cancer. 2014;24(3):394-403. Lippincott Williams and Wilkins. doi:10.1097/IGC.0000000000000062

6. Han SN, Kesic VI, Van Calsteren K, Petkovic S, Amant F. Cancer in pregnancy: a survey of current clinical practice. Eur J Obstet Gynecol Reprod Biol. 2013;167(1):18-23. doi:10.1016/j.ejogrb.2012.10.026

7. Grimm D, Woelber L, Trillsch F, Amsberg GK, Mahner S. Clinical management of epithelial ovarian cancer during pregnancy. Eur J Cancer. 2014;50(5):963-971. doi:10.1016/j.ejca.2013.12.020

8. Lockley M, Stoneham SJ, Olson TA. Ovarian cancer in adolescents and young adults. Pediatr Blood Cancer. 2019;66(3):e27512. doi: $10.1002 /$ pbc. 27512

9. Ishioka SI, Hayashi T, Endo T, Baba T, Honma H, Saito T. Advanced epithelial ovarian carcinoma during pregnancy. Int J Clin Oncol. 2007;12(5):375-378. doi:10.1007/s10147-007-0655-0

10. Minig L, Otaño L, Diaz-Padilla I, Alvarez Gallego R, Patrono MG, Valero De Bernabé J. Therapeutic management of epithelial ovarian cancer during pregnancy. Clin Transl Oncol. 2013;15(4):259-264. doi:10.1007/s12094-012-0963-3

11. Van Calsteren K, Heyns L, De Smet F, et al. Cancer during pregnancy: an analysis of 215 patients emphasizing the obstetrical and the neonatal outcomes. J Clin Oncol. 2010;28(4):683-689. doi:10.1200/ JCO.2009.23.2801

12. Morice P, Uzan C, Gouy S, Verschraegen C, Haie-Meder C. Gynaecological cancers in pregnancy. Lancet. 2012;379(9815):558-569. Lancet Publishing Group. doi:10.1016/S0140-6736(11)60829-5

13. Cavaco-Gomes J, Jorge Moreira C, Rocha A, Mota R, Paiva V, Costa A. Investigation and management of adnexal masses in pregnancy. Scientifica. 2016;2016.

14. Guidelines for diagnostic imaging during pregnancy and lactation ACOG. [Online]. Available from: https://www.acog.org/clinical/clinicalguidance/committee-opinion/articles/2017/10/guidelines-for-diagnosticimaging-during-pregnancy-and-lactation. Accessed October 08, 2020.

15. Yen CF, Lin S-L, Murk W, et al. Risk analysis of torsion and malignancy for adnexal masses during pregnancy. Fertil Steril. 2009;91(5):1895-1902. doi:10.1016/j.fertnstert.2008.02.014

16. Marret H, Lhommé C, Lecuru F, et al. Guidelines for the management of ovarian cancer during pregnancy. Eur J Obstet Gynecol Reprod Biol. 2010;149(1):18-21. Elsevier Ireland Ltd. doi:10.1016/ j.ejogrb.2009.12.001 
17. Kanal E, Barkovich AJ, Bell C, et al. ACR guidance document on MR safe practices: 2013. J Magn Reson Imaging. 2013;37 (3):501-530. doi:10.1002/jmri.24011

18. Lu J, Zheng Z, Zhang Q, et al. Measurement of HE4 and CA125 and establishment of reference intervals for the ROMA index in the sera of pregnant women. J Clin Lab Anal. 2018;32(5):e22368. doi:10.1002/jcla.22368

19. Moore RG, Miller MC, Eklund EE, Lu KH, Bast RC, LambertMesserlian G. Serum levels of the ovarian cancer biomarker HE4 are decreased in pregnancy and increase with age. Am J Obstet Gynecol. 2012;206(4):349.e1-349.e7. doi:10.1016/j.ajog.2011.12.028

20. Makkonen M, Penttilä IM, Castrén O. Serum lactic acid dehydrogenase and isoenzymes during pregnancy and labor. Acta Obstet Gynecol Scand. 1980;59(2):97-101. doi:10.3109/00016348009154622

21. Yoshimura T, Takemori K, Okazaki T, Suzuki A. Serum lactic dehydrogenase and its isoenzymes in patients with ovarian dysgerminoma. Int J Gynecol Obstet. 1988;27(3):459-465. doi:10.1016/00207292(88)90131-2

22. Horbelt D, Delmore J, Meisel R, Cho S, Roberts D, Logan D. Mixed germ cell malignancy of the ovary concurrent with pregnancy. Obstet Gynecol. 1994;4(84).

23. Zemlickis D, Lishner M, Degendorfer P, Panzarella T, Sutcliffe SB, Koren G. Fetal outcome after in utero exposure to cancer chemotherapy. Arch Intern Med. 1992;152(3):573. doi:10.1001/ archinte.1992.00400150093017

24. Cole LA. HCG variants, the growth factors which drive human malignancies. Am J Cancer Res. 2012;2(1).

25. Liu N, Peng S-M, Zhan G-X, et al. Human chorionic gonadotropin $\beta$ regulates epithelial-mesenchymal transition and metastasis in human ovarian cancer. Oncol Rep. 2017;38(3):1464-1472. doi:10.3892/or.2017.5818

26. Muttukrishna S. Role of inhibin in normal and high-risk pregnancy. Semin Reprod Med. 2004;22(3):227-234. doi:10.1055/s-2004-831898

27. Fowler PA, Evans LW, Groome NP, Templeton A, Knight PG. A longitudinal study of maternal serum inhibin-A inhibin-B, activin- $\mathrm{A}$ activin- $\mathrm{AB}$, pro- $\alpha \mathrm{C}$ and follistatin during pregnancy. Hum Reprod. 1998;13(12):3530-3536. doi:10.1093/humrep/13.12.3530

28. La Marca A, Broekmans FJ, Volpe A, Fauser BC, Macklon NS. AntiMullerian hormone (AMH): what do we still need to know? Hum Reprod. 2009;24(9):2264-2275. doi:10.1093/humrep/dep210

29. La Marca A, Giulini S, Orvieto R, De Leo V, Volpe A. AntiMüllerian hormone concentrations in maternal serum during pregnancy. Hum Reprod. 2005;20(6):1569-1572. doi:10.1093/humrep/ deh819

30. Agarwal N, Kriplani PA, Bhatla N, Gupta A. Management and outcome of pregnancies complicated with adnexal masses. Arch Gynecol Obstet. 2003;267(3):148-152. doi:10.1007/s00404-001-0287-y

31. Leiserowitz GS. Managing ovarian masses during pregnancy. Obstet Gynecol Surv. 2006;61(7):463-470. doi:10.1097/01.ogx.0000224614.5 $1356 . \mathrm{b} 7$

32. Horowitz NS. Management of adnexal masses in pregnancy. Clin Obstet Gynecol. 2011;54(4):519-527. doi:10.1097/GRF.0b013e318 $236 \mathrm{c} 583$

33. Pearl J, Price R, Richardson W, Fanelli R. Guidelines for diagnosis, treatment, and use of laparoscopy for surgical problems during pregnancy. Surg Endoscopy. 2011;25:3479-3492. doi:10.1007/ s00464-011-1927-3

34. Munnell EW. Is conservative therapy ever justified in Stage I (IA) cancer of the ovary? Am J Obstet Gynecol. 1969;103(5):641-653. doi:10.1016/0002-9378(69)90561-4

35. Kajiyama H, Shibata K, Suzuki S, et al. Fertility-sparing surgery in young women with invasive epithelial ovarian cancer. Eur J Surg Oncol. 2010;36(4):404-408. doi:10.1016/j.ejso.2010.01.005

36. Satoh T, Tsuda H, Kanato K, et al. A non-randomized confirmatory study regarding selection of fertility-sparing surgery for patients with epithelial ovarian cancer: Japan Clinical Oncology Group Study (JCOG1203). Jpn J Clin Oncol. 2015;45:6. doi:10.1093/jjco/hyv032
37. Fruscio R, Corso S, Ceppi L, et al. Conservative management of early-stage epithelial ovarian cancer: results of a large retrospective series. Ann Oncol. 2013;24(1):138-144. doi:10.1093/annonc/mds241

38. Smith HO, Berwick M, Verschraegen CF, et al. Incidence and survival rates for female malignant germ cell tumors. Obstet Gynecol. 2006;107(5):1075-1085. doi:10.1097/01.AOG.0000216004.22588.ce

39. Morice P, Denschlag D, Rodolakis A, et al. Recommendations of the fertility task force of the European Society of Gynecologic Oncology about the conservative management of ovarian malignant tumors. Int J Gynecol Cancer. 2011;21:5. doi:10.1097/IGC.0b013e3182 1 bec $6 \mathrm{~b}$

40. Satoh T, Aoki Y, Kasamatsu T, et al. Administration of standard-dose BEP regimen (bleomycin + etoposide + cisplatin) is essential for treatment of ovarian yolk sac tumour. Eur J Cancer. 2015;51 (3):340-351. doi:10.1016/j.ejca.2014.12.004

41. Chatziioannidou K, Botsikas D, Tille JC, Dubuisson J. Preservation of fertility in non-Peutz- Jegher syndrome-associated ovarian sex cord tumour. BMJ Case Rep. 2015, 2015. doi:10.1136/bcr-2014207841

42. Stavrakis T, Kalogiannidis I, Petousis S, et al. Fertility-sparing management and obstetric outcomes in a 20 -year-old patient with a Sertoli-Leydig cell tumor of the ovary: a case report and review of the literature. Oncol Lett. 2016;12:2. doi:10.3892/ol.2016.4695

43. Koren G. Fetal risks of maternal pharmacotherapy: identifying signals. Handb Exp Pharmacol. 2011;205.

44. Schaefer C. Drug safety in pregnancy-a particular challenge. Bundesgesundheitsblatt Gesundheitsforschung Gesundheitsschutz. 2018;61:9.

45. Amant F, Loibl S, Neven P, Van Calsteren K. Breast cancer in pregnancy. Lancet. 2012;379(9815):570-579. Lancet Publishing Group. doi:10.1016/S0140-6736(11)61092-1

46. Halaska MJ, Komar M, Vlk R, et al. A pilot study on peak systolic velocity monitoring of fetal anemia after administration of chemotherapy during pregnancy. Eur J Obstet Gynecol Reprod Biol. 2014;174(1):76-79. doi:10.1016/j.ejogrb.2013.12.021

47. Tetro N, Moushaev S, Rubinchik-Stern M, Eyal S. The placental barrier: the gate and the fate in drug distribution. Pharm Res. 2018;35:4. doi:10.1007/s11095-017-2286-0

48. Berveiller P, Mir O. Taxanes during pregnancy: probably safe, but still to be optimized. Oncology. 2012;83:4.

49. Cardonick E, Iacobucci A. Use of chemotherapy during human pregnancy. Lancet Oncol. 2004;5:5. doi:10.1016/S1470-2045(04) 01466-4

50. Ruiz Ramos J, Roma E, Palomar L, Poveda JL. Paclitaxel and carboplatin treatment for advanced ovarian cancer during pregnancy. Chemotherapy. 2014;59(5):344-345. doi:10.1159/ 000360691

51. Slimano F, Baudouin A, Zerbit J, et al. Cancer, immune suppression and coronavirus disease-19 (COVID-19): need to manage drug safety (French Society for Oncology Pharmacy [SFPO] guidelines). Cancer Treat Rev. 2020;88:102063. doi:10.1016/j.ctrv.2020.102063

52. Shah R, Xia C, Krailo M, et al. Is carboplatin-based chemotherapy as effective as cisplatin-based chemotherapy in the treatment of advanced-stage dysgerminoma in children, adolescents and young adults? Gynecol Oncol. 2018;150(2):253-260. doi:10.1016/j. ygyno.2018.05.025

53. Ghaemmaghami F, Abbasi F, Abadi AGN. A favorable maternal and neonatal outcome following chemotherapy with etoposide, bleomycin, and cisplatin for management of grade 3 immature teratoma of the ovary. J Gynecol Oncol. 2009;20(4):257. doi:10.3802/ jgo.2009.20.4.257

54. Cardonick E, Usmani A, Ghaffar S. Perinatal outcomes of a pregnancy complicated by cancer, including neonatal follow-up after in utero exposure to chemotherapy: results of an international registry. Am J Clin Oncol Cancer Clin Trials. 2010;33(3). 
55. Han JY, Nava-Ocampo AA, Kim TJ, Shim JU, Park CT. Pregnancy outcome after prenatal exposure to bleomycin, etoposide and cisplatin for malignant ovarian germ cell tumors: report of 2 cases. Reprod Toxicol. 2005;19(4):557-561. doi:10.1016/j.reprotox.2004.11.002

56. Amant F, Berveiller P, Boere IA, et al. Gynecologic cancers in pregnancy: guidelines based on a third international consensus meeting. Ann Oncol. 2019;30:10. doi:10.1093/annonc/mdz228

57. Alfasi A, Ben-Aharon I. Breast cancer during pregnancy - current paradigms, paths to explore. Cancers. 2019;11:11. doi:10.3390/ cancers 11111669
58. Boere I, Lok C, Vandenbroucke T, Amant F. Cancer in pregnancy: safety and efficacy of systemic therapies. Curr Opin Oncol. 2017;29:5. doi:10.1097/CCO.0000000000000386

59. Cordeiro CN, Gemignani ML. Gynecologic malignancies in pregnancy: balancing fetal risks with oncologic safety. Obstet Gynecol Surv. 2017;72(3):184-193. doi:10.1097/OGX.0000000000000407

\section{Publish your work in this journal}

Cancer Management and Research is an international, peer-reviewed open access journal focusing on cancer research and the optimal use of preventative and integrated treatment interventions to achieve improved outcomes, enhanced survival and quality of life for the cancer patient.
The manuscript management system is completely online and includes a very quick and fair peer-review system, which is all easy to use. Visit http://www.dovepress.com/testimonials.php to read real quotes from published authors. 